\title{
Lipolytic efficacy of alginate double-layer nanoemulsion containing oleoresin capsicum in differentiated 3T3-L1 adipocytes
}

\author{
Mak-Soon Lee ${ }^{\mathrm{a} *}$, Sunyoon Junga*, Yoonjin Shin ${ }^{\mathrm{a}}$, Seohyun Lee ${ }^{\mathrm{a}}$, Chong-Tai Kim ${ }^{\mathrm{b}}$, In-Hwan Kim ${ }^{\mathrm{c}}$ \\ and Yangha Kim ${ }^{\mathrm{a}}$
}

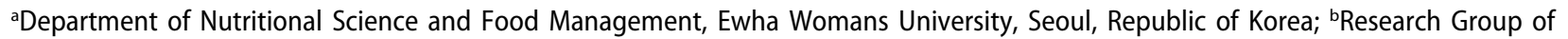
Bioprocess Engineering, Korea Food Research Institute, Seongnam, Republic of Korea; 'Department of Food and Nutrition, Korea University, Seoul, Republic of Korea
\end{abstract}

\begin{abstract}
Background: Oleoresin capsicum (OC) is an organic extract from fruits of the genus Capsicum, and has been reported to have an anti-obesity effect.

Objective: This study comparatively investigated lipolytic effects of single-layer nanoemulsion (SN) and alginate double-layer nanoemulsion (AN) containing OC in 3T3-L1 adipocytes.

Methods: SN and AN were compared by analyzing the intracellular lipid accumulation, triglyceride (TG) content, release of free fatty acids (FFAs) and glycerol, and mRNA expression of genes related to adipogenesis and lipolysis were analyzed in fully differentiated 3T3-L1 adipocytes.

Results: Compared with SN, AN exhibited higher efficiency in inhibiting the intracellular lipid accumulation and TG content, and enhanced the release of FFAs and glycerol into the medium. In AN-treated cells, mRNA levels of peroxisome proliferator-activated receptor- $\gamma$ and the fatty acidbinding protein adipocyte protein-2, which are involved in adipogenesis, were down-regulated, whereas those of genes related to lipolysis, including hormone-sensitive lipase and carnitine palmitoyl transferase-1a, were up-regulated compared with SN-treated cells.

Conclusion: The lipolytic effect of AN was greater than that of SN; this was partly associated with the increased TG hydrolysis via induction of lipolytic gene expression and suppression of adipogenic gene expression in 3T3-L1 adipocytes.
\end{abstract}

\section{ARTICLE HISTORY}

Received 22 December 2016 Accepted 19 May 2017

\section{KEYWORDS}

Free fatty acid; glycerol; lipolysis; mRNA expression; obesity

\section{Introduction}

Adipocytes play an important role in energy homeostasis. In a condition of energy excess, adipocytes store surplus energy in the form of triglyceride (TG). within the cell, which can lead to obesity [1]. Given that obesity is highly associated with several metabolic diseases such as diabetes mellitus, cardiovascular diseases, and certain types of cancers [2], it is important for researchers to develop new compounds that effectively catabolize lipids accumulated in the adipocytes.

Oleoresin capsicum (OC) is an organic solvent extract from dried ripe fruits of the genus Capsicum [3]. It is widely used as a food additive to enhance the taste and shelf-life of food products [4]. Capsaicinoids contribute to the pungent flavor of the OC, and capsaicin is the main secondary metabolite in capsaicinoid present in red pepper [5]. The red pepper extract has shown beneficial effects on cancer [6], oxidative stress [7], and inflammation [7]. Furthermore, it has been shown that red pepper extract and capsaicin have anti-obesity effects in vitro $[1,8]$ and in vivo $[9,10]$. In particular, an ethanol extract of red pepper seed was found to decrease glycerol3-phosphate dehydrogenase (GPDH) activity and CCAAT-enhancer-binding protein- $\alpha$ (C/EBP)- $\alpha$ and C/EBP- $\beta$ messenger RNA (mRNA) expression in 3T3L1 adipocytes [11]. Capsicum annuum L. water extracts were found to inhibit lipoprotein lipase (LPL) activity and mRNA expression in 3T3-L1 cells [8]. In addition, capsaicin reduced the body weight gain and visceral adipose tissue mass in mice fed a high-fat diet [9] and decreased the fasting glucose/insulin and TG levels in the plasma of genetically obese/diabetic mice [10].

Nanoemulsions are oil-in-water emulsions with mean droplet diameters ranging from 20 to $500 \mathrm{~nm}$ [12]. Nanoemulsion technology has been successfully used in the food industry to encapsulate food components such as capsaicin and resveratrol in nanometer-

CONTACT Yangha Kim yhmoon@ewha.ac.kr Department of Nutritional Science and Food Management, Ewha Womans University, 52, Ewhayeodae-gil, Seodaemun-gu, Seoul, 03760, Korea

*These authors contributed equally to this work.

(4) Supplemental data for this article can be accessed here.

( 2017 The Author(s). Published by Informa UK Limited, trading as Taylor \& Francis Group.

This is an Open Access article distributed under the terms of the Creative Commons Attribution License (http://creativecommons.org/licenses/by/4.0/), which permits unrestricted use, distribution, and reproduction in any medium, provided the original work is properly cited. 


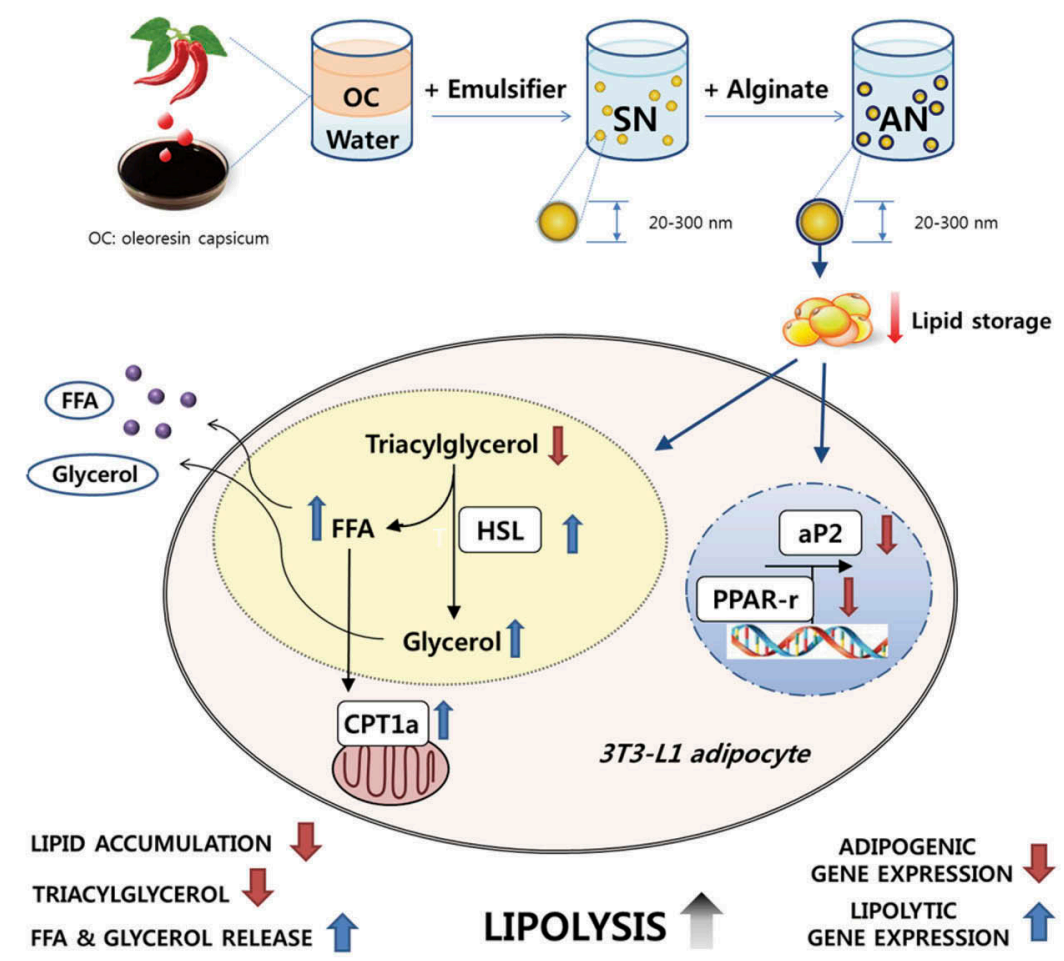

Figure 1. Schematic representation of a hypothesis on the efficacy of alginate double-layer nanoemulsion (AN) in 3T3-L1 adipocytes. OC, oleoresin capsicum; SN, single-layer nanoemulsion; FFA, free fatty acid; HSL, hormone-sensitive lipase; CPT1a, carnitine palmitoyl transferase-1a; aP2, adipocyte protein-2; PPAR-r, peroxisome proliferator-activated receptor- $\gamma$.

sized structures [13]. The potential benefits of nanoemulsions include enhanced stability and oral bioavailability of hydrophobic functional food ingredients [14]. Choi et al. developed an OC-loaded double-layered nanoemulsion using a self-assembly emulsification method [13]. The stability and physicochemical properties of the double-layer nanoemulsion were enhanced compared with those of single-layered nanoemulsions, which implies that the double-layered nanoemulsion can be used in the production of functional foods containing lipophilic bioactive ingredients. Previously, we conducted an in vivo experiment by comparing the anti-obesity effects of OC and nanoemulsion oleoresin capsicum (NOC) [15]. NOC was found to be more effective than OC in inhibiting body weight gain and adipogenic gene expression in rats fed a high-fat diet. However, the detailed mechanism of the lipolytic effect of NOC involved in the anti-obesity effect remains unclear. In addition, studies comparing the lipolytic efficiency of the both single- and double-layered nanoemulsions have not been reported.

We hypothesized that a double-layer nanoemulsion containing OC (AN), which is coated with a natural biopolymer alginate, could have greater efficacy on lipolysis in 3T3-L1 adipocytes compared with a single-layer nanoemulsion containing $\mathrm{OC}(\mathrm{SN})$, as schematically presented in Figure 1. Therefore, we evaluated intracellular lipid accumulation and TG level, and the release of free fatty acids (FFAs) and glycerol under SN or AN treatment in 3T3-L1 adipocytes. In addition, the expression of genes related to adipogenesis, such as peroxisome proliferatoractivated receptor- $\gamma$ (PPAR- $\gamma$ ) and the fatty acid-binding protein adipocyte protein-2 (aP2), was compared. The mRNA levels of hormone-sensitive lipase (HSL) and carnitine palmitoyltransferase-1a (CPT-1 $\alpha$ ), involved in lipolysis, were also compared.

\section{Materials and methods}

\section{Cells and reagents}

The 3T3-L1 cell line was obtained from the American Type Culture Collection (Manassas, VA, USA). OC was supplied by General Foods and Flavors (Seoul, Korea). A $0.45 \mu \mathrm{m}$ polyvinylidene fluoride (PVDF) membrane filter was purchased from Whatman (Maidstone, UK). Dulbecco's modified Eagle's medium (DMEM), glutamine, penicillin-streptomycin, fetal bovine serum (FBS), and TRIzol reagent were obtained from Invitrogen (Carlsbad, CA, USA). A cell count kit-8 (CCK-8) was purchased from Dojindo Laboratories (Kumamoto, Japan). Nonidet P-40 was purchased from Sigma-Aldrich (Saint Louis, MO, 
USA). Assay kits for TG were purchased from Asan Pharmaceuticals (Seoul, Korea). Moloney murine leukemia virus (M-MLV) reverse transcriptase was purchased from Promega (Madison, WI, USA). Universal SYBR ${ }^{\circ}$ Green Master mix for quantitative polymerase chain reaction (qPCR) was purchased from Qiagen (Chatsworth, CA, USA). Assay kits for non-esterified FFAs and glycerol were obtained from Wako (Osaka, Japan) and Roche Molecular Biochemicals (Mannheim, Germany), respectively. A bicinchoninic acid (BCA) protein assay kit was obtained from Thermo Scientific (Pittsburgh, PA, USA).

\section{Preparation of SN and AN}

Samples (SN and AN) were kindly supplied by the Korea Food Research Institute (Seongnam, Gyeonggi, Korea) [13]. Nanoemulsions were prepared using the self-assembly method and classified into two groups: $\mathrm{SN}$ based on the aqueous phase with water and AN based on alginate. In brief, the $\mathrm{SN}$ and $\mathrm{AN}$ were prepared using Tween 80 , which was selected as the optimum detergent for the formation of oil-in-water $(\mathrm{O} /$ W) NOC by a pseudoternary phase diagram. The OC and Tween $80(0.5 \mathrm{~g})$ were mixed in the ratio of 1:3. The mixture was dissolved at $25^{\circ} \mathrm{C}$ for $2 \mathrm{~h}$ with constant stirring in $100 \mathrm{~mL}$ of water (SN) or alginate $(0.5 \%, \mathrm{w} / \mathrm{v})$ solution $(\mathrm{AN})$ and then filtered using a $0.45 \mu \mathrm{m}$ PVDF membrane filter. The filtrates were stabilized for $24 \mathrm{~h}$ at $25^{\circ} \mathrm{C}$. The amounts of capsaicin in the $\mathrm{SN}$ and $\mathrm{AN}$ were quantified by high-performance liquid chromatography, and were prepared using the same concentration $(5.1 \mu \mathrm{g} / \mathrm{g})$.

\section{Cell culture and differentiation}

The 3T3-L1 fibroblasts were maintained initially in DMEM supplemented with $10 \%(\mathrm{v} / \mathrm{v}) \mathrm{FBS}, 2 \mathrm{mmol} / \mathrm{L}$ glutamine, 100 units $/ \mathrm{mL}$ penicillin, and $100 \mu \mathrm{g} / \mathrm{mL}$ streptomycin at $37^{\circ} \mathrm{C}$ and $5 \%$ carbon dioxide. To induce adipocyte differentiation, 3T3-L1 cells were grown to confluence and cultured with a differentiation medium containing $0.5 \mathrm{mmol} / \mathrm{L}$ 3-isobutyl-1-methylxanthine, $1 \mu \mathrm{mol} / \mathrm{L}$ dexamethasone, and $5 \mu \mathrm{g} / \mathrm{mL}$ insulin (MDI). After $48 \mathrm{~h}$ of exposure to the differentiation medium, the cells were maintained for an additional 7 days in DMEM supplemented with 10\% FBS. Fully differentiated 3T3-L1 adipocytes in serum-free medium containing $2 \%$ bovine serum albumin (BSA) were treated with indicated concentrations of $\mathrm{SN}$ or AN for $24 \mathrm{~h}$. All the measurements were performed in triplicate.

\section{Cytotoxicity assay}

Cell viability was determined as described previously [16] using a CCK-8 kit according to the manufacturer's instructions. Differentiated 3T3-L1 adipocytes were treated with 0 (control), $0.1,1,10,100$, or $1000 \mathrm{ng} / \mathrm{mL}$ of SN or $\mathrm{AN}$ for 4 and $24 \mathrm{~h}$ at $37^{\circ} \mathrm{C}$. For the negative and positive control samples, $0.1 \%$ dextran and $0.1 \%$ sodium dodecyl sulfate (SDS) was added, respectively. Absorbance was measured using a Varioskan plate reader at $450 \mathrm{~nm}$ (Thermo Electron, Waltham, MA, USA) and results are presented as the percentage of untreated control cells. All measurements were performed in at least three independent experiments, each of which was performed in triplicate $(n=3)$.

\section{Oil Red $O$ staining}

Lipid accumulation was measured as previously described [16]. In brief, fully differentiated 3T3-L1 adipocytes were maintained in serum-free medium containing $2 \%$ BSA with $0,1,10,100$, or $1000 \mathrm{ng} / \mathrm{mL}$ of SN or AN for $24 \mathrm{~h}$. The cells were washed with phosphate-buffered saline (PBS; $\mathrm{pH}$ 7.4) and fixed with $10 \%(\mathrm{v} / \mathrm{v})$ formalin in PBS. The accumulation of lipid was measured by staining the cells with Oil Red $\mathrm{O}$ (saturated Oil Red O dye in six parts isopropanol and four parts water) for $15 \mathrm{~min}$. Quantification of the stain was carried out by dissolving the stained oil droplets in the cell monolayer with $4 \%(\mathrm{v} / \mathrm{v})$ Nonidet P-40 in isopropanol, followed by measuring the absorbance at $520 \mathrm{~nm}$. Values are presented as the percentage of control cells treated without $\mathrm{SN}$ or AN.

\section{TG assay}

For the measurement of intracellular TG, a TG assay kit was used according to the method described previously [16]. Differentiated 3T3-L1 adipocytes were lysed in a lysis buffer that consisted of $1 \%$ Triton $\mathrm{X}-100$ in PBS, and the cellular content of TG was determined using a commercial TG assay kit. The cellular TG content was then normalized to the protein concentration measured by a BCA protein assay kit.

\section{FFA and glycerol assay}

To investigate the concentration-dependent effects, differentiated 3T3-L1 adipocytes were treated in serum-free medium containing $2 \%$ BSA with 0 (control), 1, 10, 100 , or $1000 \mathrm{ng} / \mathrm{mL}$ of SN or AN for $24 \mathrm{~h}$. To investigate the concentration-dependent effects, $1000 \mathrm{ng} / \mathrm{mL}$ of SN or AN was treated for $1,3,6,16$, and $24 \mathrm{~h}$. Medium was 
collected from the culture plate and heated at $65^{\circ} \mathrm{C}$ for $8 \mathrm{~min}$ to inactivate any enzymes released from the cells. The amounts of FFAs and glycerol released into the medium were measured using colorimetric enzyme assay kits in accordance with the manufacturer's instructions. The cellular protein concentration was determined using a BCA protein assay kit. The glycerol and FFA concentrations were normalized to the cellular protein content.

\section{Real-time $q P C R$}

Total RNA was extracted from 3T3-L1 adipocytes using TRIzol Reagent. Complementary DNA (cDNA) was synthesized from $4 \mu \mathrm{g}$ RNA using M-MLV reverse transcriptase. qPCR was then performed in $25 \mu \mathrm{L}$ Universal SYBR Green Master mix using a fluorometric thermal cycler (Rotor-Gene ${ }^{\text {TM }}$ 2000; Corbett Research, Mortlake, NSW, Australia). The reaction mixtures were incubated for initial denaturation at $95^{\circ} \mathrm{C}$ for 15 min, followed by 50 cycles of PCR. Each cycle included denaturation at $95^{\circ} \mathrm{C}$ for $15 \mathrm{~s}$, annealing at $55^{\circ} \mathrm{C}$ for $20 \mathrm{~s}$, and primer extension at $72^{\circ} \mathrm{C}$ for $20 \mathrm{~s}$. Primers were designed using an online program (primer3_http://www.cgivo.2) [17]. Sequences of the sense and antisense primers used for amplification were: PPAR- $\gamma, \quad 5^{\prime}$-TGTGGGGATAAAGCATCAGC-3' and 5'-CAAGGCACTTCTGAAACCGA-3'; aP2, 5'TCACCCCAGATGACAGGAAA- $3^{\prime}$ and $5^{\prime}$ CATGACACATTCCACCACCA-3'; HSL, 5' ACTCAGACCAGAAGGCACTA-3' and 5'TAGTTCCAGGAAGGAGTTGA-3'; CPT- $1 \alpha, \quad 5^{\prime}-$ CTGTTGGAGGTGACAGACTT-3' and $3^{\prime}$ CACTTTCTCTTTCCACAAGG-3'; and $\beta$-actin, $5^{\prime}$ GTTGCCAATAGTGATGACCT- $3^{\prime}$ and $5^{\prime}$ GGACCTGACAGACTACCTCA- $3^{\prime}$. The ${ }^{\Delta \Delta C}{ }_{T}$ method was used for relative quantification, and its value for each sample was determined by calculating the difference between the $C_{T}$ value of the target gene and the $C_{T}$ value of the $\beta$-actin reference gene. The normalized level of expression of the target gene in each sample was calculated using the formula $2^{-\Delta \Delta C}$. Values were expressed as a fold of the control.

\section{Statistical analysis}

Values are expressed as mean \pm SE. Statistical analyses were performed using SPSS software (version 19; IBM Corp., Armonk, NY, USA). The significance of differences between two groups of SN and AN at the same concentration were determined with a Student's $t$ test (two-tailed). Significant differences among different concentrations of treatment group were analyzed using a one-way analysis of variance (ANOVA), followed by Tukey's multiple comparison tests. A value of $p<0.05$ indicated a significant difference.

\section{Results}

\section{Effects of SN and AN on viability of 3T3-L1 adipocytes}

Potential cytotoxic effects of SN and AN on 3T3-L1 adipocytes were investigated. 3T3-L1 adipocytes were treated with 0 (control), 0.1, 1, 10, 100, and $1000 \mathrm{ng} /$ $\mathrm{mL} \mathrm{SN}$ or $\mathrm{AN}$, and incubated for 4 or $24 \mathrm{~h}$. Cell viability of $\mathrm{SN}, \mathrm{AN}$, or $0.1 \%$ dextran negative control (NC) remained unchanged at various concentrations after 4 and $24 \mathrm{~h}$ of incubation (supplementary Figure $\mathrm{S} 1$ ), and the optical density (OD) ranged from 0.83 to 1.30. However, in the positive control (PC) with $0.1 \%$ SDS, cell viability was quite apparently decreased by 93.1-93.7\% (0.03-0.08 OD) after $4 \mathrm{~h}$ of incubation, compared to untreated control.

\section{Effects of $S N$ and $A N$ on intracellular lipid accumulation and TG content in 3T3-L1 adipocytes}

To evaluate the effects of SN and AN on intracellular lipid accumulation and TG contents, fully differentiated adipocytes were treated with 0 (control), 1, 10, 100 , and $1000 \mathrm{ng} / \mathrm{mL}$ of $\mathrm{SN}$ or AN for $24 \mathrm{~h}$. The intracellular lipid content decreased in a concentration-dependent manner by $15 \%$ and $20 \%$ in 100 and $1000 \mathrm{ng} / \mathrm{mL}$ of AN, respectively, compared with that of the control (Figure 2(b)). Furthermore, the intracellular lipid content of AN was found to be lower than that of SN by $12.1 \%$ in $1000 \mathrm{ng} / \mathrm{mL}$ (Figure 2(a,b)). The TG content decreased by $11 \%$ and $21 \%$ in 100 and $1000 \mathrm{ng} /$ $\mathrm{mL}$ of $\mathrm{AN}$, respectively, compared with that of the control (Figure 2(c)). On the other hand, there were no significant differences in the presence of $\mathrm{SN}$ (Figure 2(c)).

\section{Effects of SN and AN on FFA and glycerol concentration in 3T3-L1 adipocytes}

Lipolytic effects of the SN and AN on fat disintegration were measured by estimating the amounts of FFA and glycerol released in the medium of adipocytes. The effect of SN and AN on FFA and glycerol was both concentration and time dependent. First, to investigate the concentration-dependent effects, the differentiated 3T3-L1 adipocytes were treated with various concentrations [0 (control), $0.1,1,10,100$, and $1000 \mathrm{ng} / \mathrm{mL}$ ] of $\mathrm{SN}$ and $\mathrm{AN}$ for $24 \mathrm{~h}$. AN 
(A)
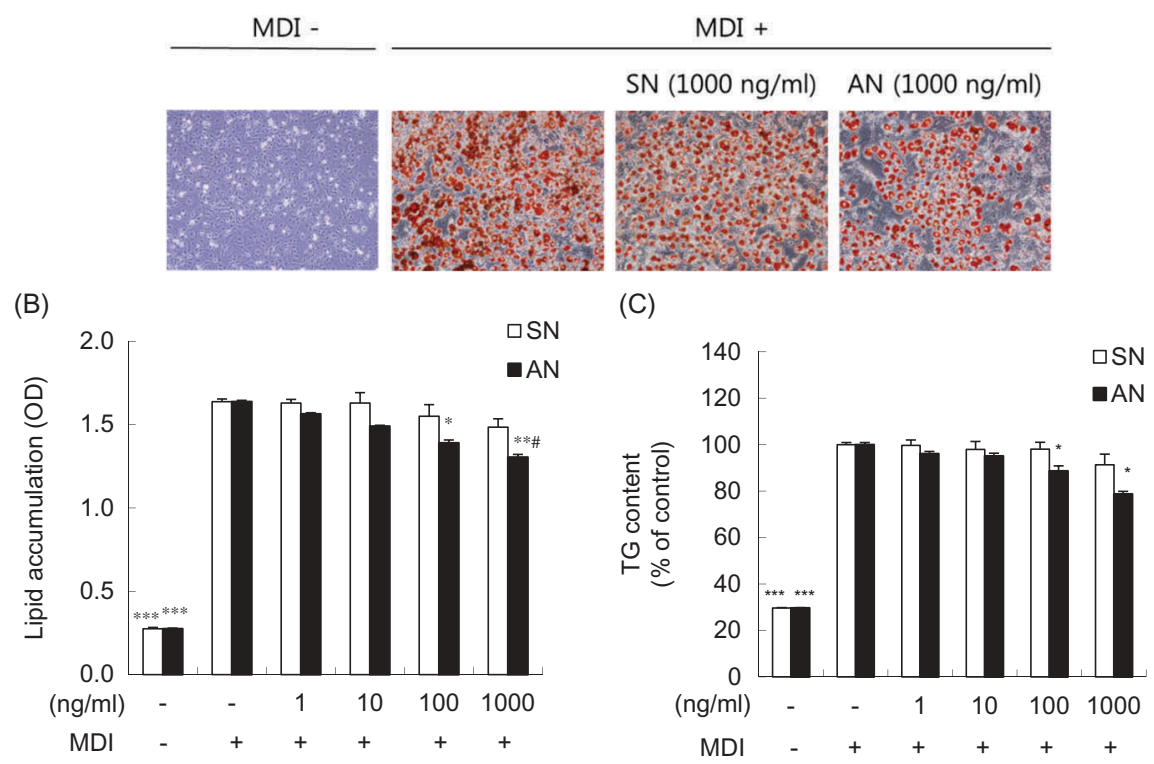

Figure 2. Effects of single-layer nanoemulsion (SN) and alginate double-layer nanoemulsion (AN) on the lipid accumulation and triglyceride (TG) content in 3T3-L1 adipocytes. (a) The effect of SN and AN on lipid droplet formation was measured by Oil Red 0 staining. Differentiated 3T3-L1 adipocytes were treated with $1000 \mathrm{ng} / \mathrm{mL}$ of SN or AN for $24 \mathrm{~h}$. Representative cell images were captured at 100x magnification. MDI, differentiation medium containing 3-isobutyl-1-methylxanthine, dexamethasone, and insulin. Quantification of (b) intracellular lipid accumulation and (c) TG content. Differentiated 3T3-L1 adipocytes were treated with 0 (MDI treated control), 1, 10, 100, or $1000 \mathrm{ng} / \mathrm{mL}$ of SN or AN for $24 \mathrm{~h}$. Oil Red O-stained lipids were extracted in absolute isopropanol, after which the absorbance of the solution was measured at $520 \mathrm{~nm}$. TG content was determined using enzymic colorimetric methods. OD, optical density. Data are expressed as the mean \pm SE of at least three independent experiments, each performed in triplicate $(n=3)$. One-way ANOVA followed by Tukey's multiple comparison tests: ${ }^{*} p<0.05,{ }^{* *} p<0.01$, and ${ }^{* * *} p<0.001$ compared with MDI treated control group. Student's $t$ test: \#p $<0.05$ compared with SN group.

significantly increased the amount of FFA and glycerol released into the medium at $1000 \mathrm{ng} / \mathrm{mL}$, by $30 \%$ and $45 \%$, respectively, compared with the control. However, there were no significant differences in the presence of SN (Figure 3). In addition, the amounts of FFA and glycerol were significantly higher (by $23 \%$ and $26 \%$, respectively) in the $\mathrm{AN}$ treated than in the SN-treated 3T3-L1 adipocytes (Figure 3). To investigate the time-dependent effects, we treated the differentiated 3T3-L1 adipocytes with
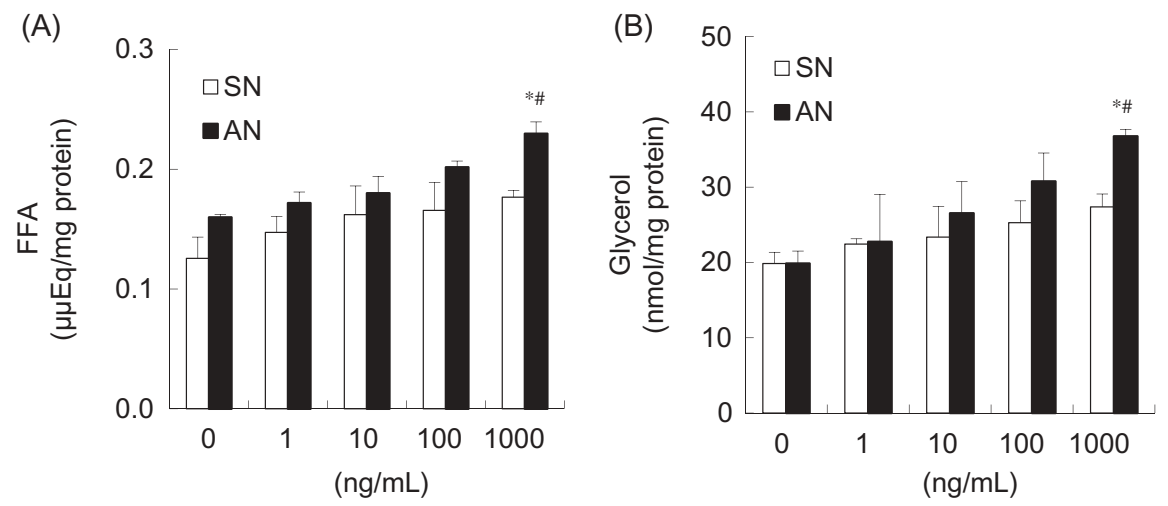

Figure 3. Concentration-dependent effects of single-layer nanoemulsion (SN) and alginate double-layer nanoemulsion (AN) on (a) free fatty acids (FFA) and (b) glycerol from adipocytes. Differentiated 3T3-L1 adipocytes were treated with 0 (untreated control), 1, 10,100 , or $1000 \mathrm{ng} / \mathrm{mL}$ of SN or AN for $24 \mathrm{~h}$. The culture medium was collected and assayed for the FFA and glycerol content. Data are expressed as the mean \pm SE of at least three independent experiments, each performed in triplicate $(n=3)$. One-way ANOVA followed by Tukey's multiple comparison tests: ${ }^{*} p<0.05$ compared with untreated control group. Student's $t$ test: \#p $<0.05$ compared with SN group. 
$1000 \mathrm{ng} / \mathrm{mL}$ of SN or AN for $1,3,6,16$, and $24 \mathrm{~h}$. The amounts of FFA and glycerol tended to increase over time with the treatment of $\mathrm{SN}$ and $\mathrm{AN}$. Specifically, after $24 \mathrm{~h}$ of incubation, the amounts of FFA and glycerol released into the medium were higher by $24.9 \%$ and $26.0 \%$, respectively, when treated with AN than with SN (Figure 4).

\section{Effects of SN and AN on gene expression in 3T3-L1 adipocytes}

We evaluated whether SN or AN stimulates lipid catabolism by regulating genes involved in adipocyte differentiation and lipolysis. AN was found to down- regulate mRNA levels of adipogenic genes such as PPAR- $\gamma$ and aP2 in a concentration-dependent manner compared with those of the control; however, SN did not exhibit any such effect (Figure 5). The mRNA levels of PPAR- $\gamma$ and aP2 were significantly lower at $1000 \mathrm{ng} / \mathrm{mL}$ of $\mathrm{AN}$ than $\mathrm{SN}$, by $38.7 \%$ and $42.4 \%$, respectively (Figure 5). The mRNA levels of lipolytic genes such as HSL and CPT-1a were upregulated by $\mathrm{AN}$ in a concentration-dependent manner compared with those of the control; however, no such effect was shown by SN (Figure 6). Specifically, the mRNA levels of HSL and CPT-1a were significantly higher at $1000 \mathrm{ng} / \mathrm{mL}$ of $\mathrm{AN}$ than $\mathrm{SN}$, by $43.2 \%$ and $33.1 \%$, respectively (Figure 6).
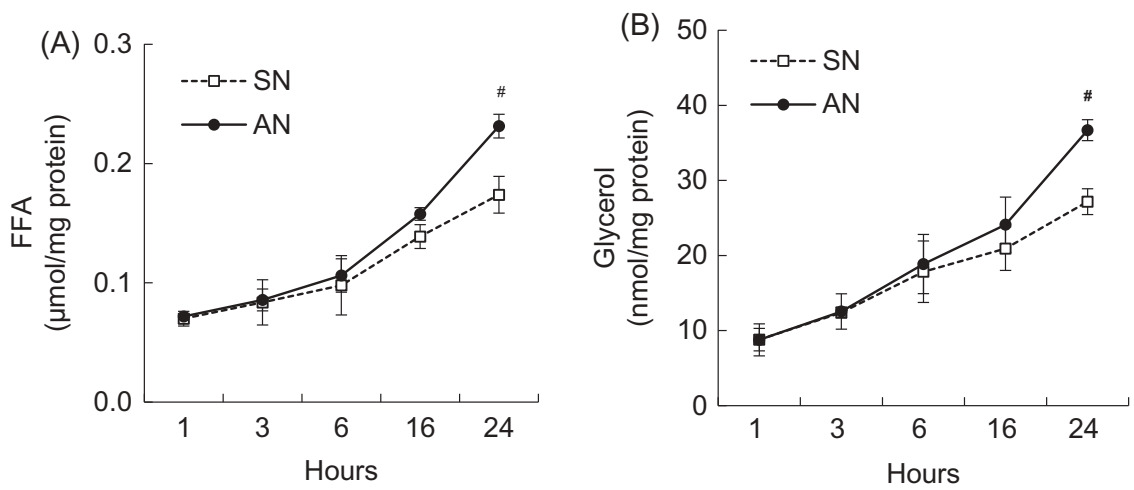

Figure 4. Time-dependent effects of single-layer nanoemulsion (SN) and alginate double-layer nanoemulsion (AN) on (a) free fatty acids (FFA) and (b) glycerol from adipocytes. Differentiated 3T3-L1 adipocytes were treated with $1000 \mathrm{ng} / \mathrm{mL}$ of SN or AN for 1, 3, 6, 16 , or $24 \mathrm{~h}$. The culture medium was collected and assayed for the FFA and glycerol content. Data are expressed as the mean \pm SE of at least three independent experiments, each performed in triplicate $(n=3)$. Student's $t$ test: \#p $<0.05$ compared with SN group.
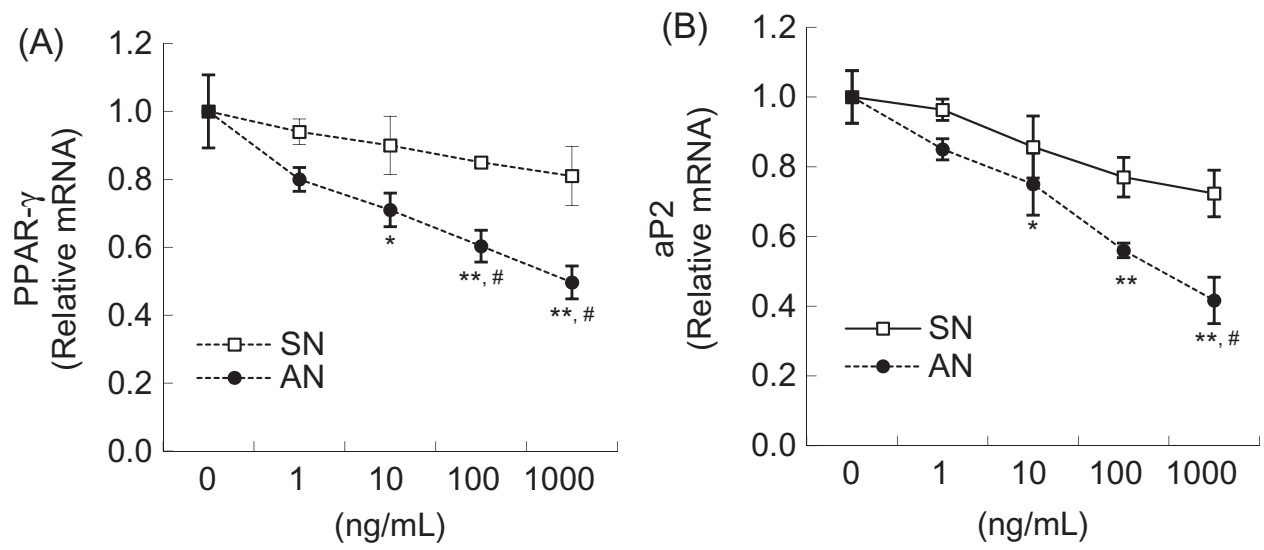

Figure 5. Effects of single-layer nanoemulsion (SN) and alginate double-layer nanoemulsion (AN) on the mRNA expression of adipogenic genes including (a) peroxisome proliferator-activated receptor- $\gamma$ (PPAR- $\gamma$ ) and (b) adipocyte protein-2 (aP2) in adipocytes. Differentiated 3T3-L1 adipocytes were treated with 0 (untreated control), 1, 10, 100, or $1000 \mathrm{ng} / \mathrm{mL}$ of SN or AN for $24 \mathrm{~h}$. The mRNA level was measured using quantitative polymerase chain reaction. The values were calculated as fold-change over the control. Data are expressed as the mean \pm SE of at least three independent experiments, each performed in triplicate $(n=3)$. Oneway ANOVA followed by Tukey's multiple comparison tests: ${ }^{*} p<0.05$ and ${ }^{* *} p<0.01$ compared with untreated control group. Student's $t$ test: \#p < 0.05 compared with SN group. 
(A)

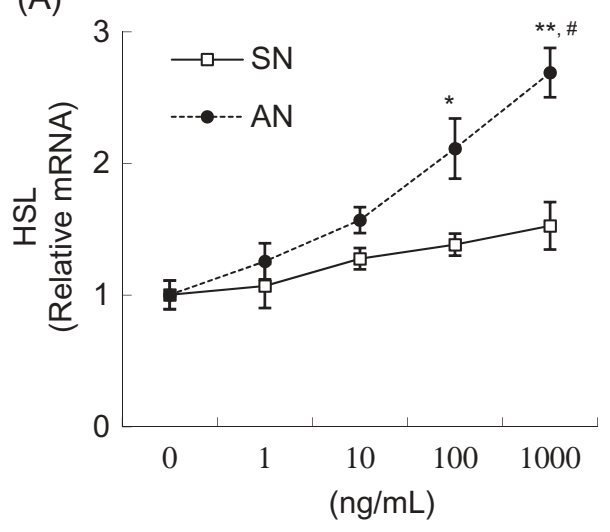

(B)

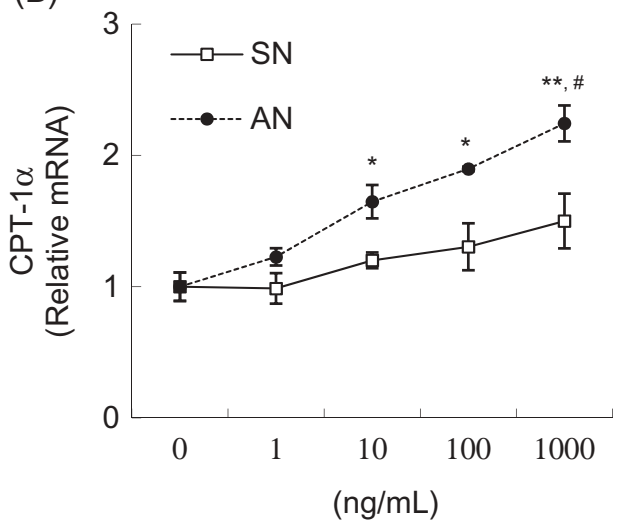

Figure 6. Effects of single-layer nanoemulsion (SN) and alginate double-layer nanoemulsion (AN) on the mRNA expression of lipolytic genes including (a) hormone-sensitive lipase (HSL) and (b) carnitine palmitoyl transferase-1a (CPT-1a) in adipocytes. Differentiated 3T3-L1 adipocytes were treated with 0 (untreated control), 1, 10, 100, or $1000 \mathrm{ng} / \mathrm{mL}$ of SN or AN for $24 \mathrm{~h}$. The mRNA level was measured using quantitative polymerase chain reaction. The values were calculated as fold-change over the control. Data are expressed as the mean \pm SE of at least three independent experiments, each performed in triplicate $(n=3)$. One-way ANOVA followed by Tukey's multiple comparison tests: ${ }^{*} p<0.05$ and ${ }^{* *} p<0.01$ compared with untreated control group. Student's $t$ test: $\# p<0.05$ compared with SN group.

\section{Discussion}

Food scientists have been striving to discover better food products with health benefits. Nanoemulsion delivery systems have been known to enhance the bioavailability of insoluble functional food ingredients [14]. In a previous study, Choi et al. established optimal conditions for the production of a stable nanoemulsion containing OC and developed $\mathrm{SN}$ and $\mathrm{AN}$, which were all used in our study $[13,18]$. The $\mathrm{SN}$ is a single-layer nanoemulsion including OC, while the AN is a double-layer nanoemulsion developed to increase the stability and physicochemical properties of the SN. The AN showed a more compact and clearer particle structure than the $\mathrm{SN}$, and was found to have higher zeta potential value, which is an indicator of the greater overall stability of the nanoemulsion compared to the SN [13]. In a 2014 study, we reported that NOCs have beneficial effects against obesity compared with OC in reducing fat mass and adipogenic gene expression in obese rats fed a high-fat diet [15]. In this study, we focused on the lipolytic effects of $\mathrm{SN}$ and $\mathrm{AN}$ in differentiated 3T3-L1 adipocytes.

To evaluate the inhibitory effects of the $\mathrm{SN}$ and $\mathrm{AN}$ on fat accumulation, the content of intracellular lipid and TG in 3T3-L1 adipocytes was measured. The current study showed that AN decreased intracellular lipid and $\mathrm{TG}$, in a concentration-dependent manner. This result was consistent with our previous study reporting that NOC had anti-obesity effects that lowered white adipose tissue mass in rats fed a high-fat diet [15]. Here, we compared the bioefficacy of two types of NOC ( $\mathrm{SN}$ and
$\mathrm{AN}$ ), and demonstrated that $\mathrm{AN}$ may be a more effective material to lower lipid accumulation than SN. Because a double-layer nanoemulsion incorporated with alginate has been reported to improve the stability of a singlelayer nanoemulsion [13], we assume that improved stability of the bioactive ingredients in AN, compared with $\mathrm{SN}$, may enhance the bioefficacy of the AN formulation.

In lipolysis, TG is broken down into FFAs and glycerol. To estimate the direct effect of $\mathrm{SN}$ and $\mathrm{AN}$ on lipolysis, the amount of FFAs and glycerol released into the medium was measured in 3T3-L1 adipocytes. In our previous study, capsaicin was shown to increase the release of FFAs and glycerol [16]. Here, AN tended to enhance the release of FFAs and glycerol in a concentration- and time-dependent manner; however, such an effect was not exhibited by SN. Furthermore, AN was more effective than $\mathrm{SN}$ in stimulating fat hydrolysis in mature adipocytes, as shown by higher amounts of FFAs and glycerol released after AN treatment than SN treatment. Since there were no data focusing on lipolytic effects of the NOC, this study suggests possible mechanisms for explaining the anti-obesity effect of NOC. In addition, it can be assumed that AN may have better lipolytic effects than $\mathrm{SN}$, which were modulated by enhancing the hydrolysis of TG into glycerol and FFAs in adipocytes.

Adipogenesis is the process by which preadipocytes become mature adipocytes. The differentiation and proliferation of preadipocytes are regulated and coordinated by several adipogenic molecules, such as PPAR- $\gamma$ and aP2 [19]. PPAR- $\gamma$ is a key transcriptional 
factor expressed at high levels in adipocytes [20]. When the expression of PPAR- $\gamma$ is activated, lipid biosynthesis pathways are stimulated through target gene expression such as C/EBP- $\alpha$ and aP2 [19,21]. The latter (aP2) is a carrier protein for fatty acids and is highly expressed during adipocyte differentiation [22]. Circulating aP2 levels increase in both genetically and diet-induced obese mice, and reducing the circulating aP2 levels to normal improves metabolic parameters in obese mice [23]. Feng et al. reported that yellow capsicum extract inhibits the expression of PPAR- $\gamma$ and C/EBP- $\alpha$ in 3T3-L1 adipocytes [24]. Furthermore, OC and NOC extracts were reported to decrease the mRNA levels of adipogenic genes including PPAR- $\gamma$, sterol regulatory element binding protein-1c (SREBP1c), and aP2 in white adipose tissue of obese rats fed a high-fat diet [15]. In this study, we found that AN reduced mRNA levels of PPAR- $\gamma$ and aP2 in a concentration-dependent manner. Specifically, AN significantly lowered mRNA levels of PPAR- $\gamma$ and aP2 compared with SN, which implies greater efficacy of AN in inhibiting adipogenic gene expression.

To evaluate the lipolytic mechanisms of the $\mathrm{SN}$ and AN, the mRNA levels of CPT-1a and HSL were evaluated. HSL is a key enzyme for the regulation of lipid storage. Upon lipolytic stimulation, HSL is activated, which hydrolyzes triacylglycerol to monoacylglycerol and FFAs [25]. Fatty acyl-coenzyme A (CoA) is generated from FFAs in the cytoplasm by fatty acyl-CoA synthase. As a result, CPT-1 $\alpha$, located on the outer mitochondrial membrane, catalyzes the formation of fatty acyl-carnitine from fatty acyl-CoA and carnitine. The acyl-carnitine complexes are then transported across the inner mitochondrial membrane to be oxidized [26]. Therefore, CPT-1a serves as a rate-limiting enzyme for fatty acid oxidation and is indicative of lipolysis. Studies have demonstrated that red pepper and capsaicin are effective in inducing lipolysis by regulating genes involved in lipid catabolism [16,27]. Capsaicin up-regulates the expression of HSL, CPT-1a, and uncoupling protein-2 (UCP2) in adipocytes [16]. Besides, Kochujang (Korean fermented red pepper paste) extract up-regulates HSL transcriptional activity in 3T3-L1 adipocytes [27]. In our study, AN increased the expression of HSL and CPT-1 $\alpha$ in a concentration-dependent manner; however, no such effect was exhibited by SN. Moreover, AN was more efficient than SN at increasing mRNA levels of HSL and CPT-1a. These results indicate that the lipolytic action of $\mathrm{AN}$ could be partly associated with the expression of genes related to lipolytic pathways, including HSL and CPT- $1 \alpha$.

In conclusion, the lipolytic activity of AN was found to be higher than that of SN, leading to reduced intercellular lipid and TG concentrations and increased hydrolysis of
TG to release FFAs and glycerol into the medium of adipocytes. The lipolytic effects are mediated, at least in part, by regulation of the expression of genes involved in the lipolytic pathway, such as HSL and CPT- $1 \alpha$, and those involved in adipogenesis, such as PPAR- $\gamma$ and aP2 expression in adipocytes. Thus, AN may be useful as a potent lipolytic agent for treating obesity.

\section{Disclosure statement}

No potential conflict of interest was reported by the authors.

\section{Funding}

This work was supported by the Korea Food Research Institute (project no. E0111300 and E0155301-03) and the National Research Foundation of Korea (NRF) grant funded by the Korea government [2016R1A2B4011021].

\section{References}

[1] Ntambi JM, Kim YC. Adipocyte differentiation and gene expression. J Nutr. 2000;130:3122S-6S.

[2] Hill JO, Peters JC. Environmental contributions to the obesity epidemic. Science. 1998;280:1371-1374.

[3] Haas JS, Whipple RE, Grant PM, et al. Chemical and elemental comparison of two formulations of oleoresin capsicum. Sci Justice. 1997;37:15-24.

[4] Eissa HA, Mostafa B, Hussein A. Capsaicin content and quality characteristics in different local pepper varieties (Capsicum annum) and acid-brine pasteurized puree. J Food Technol. 2007;5:246-255.

[5] Srinivasan K. Biological activities of red pepper (Capsicum annuum) and its pungent principle capsaicin: a review. Crit Rev Food Sci Nutr. 2015;56:1488-1500.

[6] Dwivedi V, Shrivastava R, Hussain S, et al. Cytotoxic potential of Indian spices (extracts) against esophageal squamous carcinoma cells. Asian Pac J Cancer Prev. 2011;12:2069-2073.

[7] Zimmer AR, Leonardi B, Miron D, et al. Antioxidant and anti-inflammatory properties of Capsicum baccatum: from traditional use to scientific approach. J Ethnopharmacol. 2012;139:228-233.

[8] Baek J, Lee J, Kim K, et al. Inhibitory effects of Capsicum annuum L. water extracts on lipoprotein lipase activity in 3T3-L1 cells. Nutr Res Pract. 2013;7:96-102.

[9] Lee GR, Shin MK, Yoon DJ, et al. Topical application of capsaicin reduces visceral adipose fat by affecting adipokine levels in high-fat diet-induced obese mice. Obesity. 2013;21:115-122.

[10] Kang JH, Tsuyoshi G, Le Ngoc H, et al. Dietary capsaicin attenuates metabolic dysregulation in genetically obese diabetic mice. J Med Food. 2011;14:310-315.

[11] Jeon G, Choi Y, Lee SM, et al. Anti-obesity activity of methanol extract from hot pepper (Capsicum annuum L.) seeds in 3T3-L1 adipocyte. Food Sci Biotechnol. 2010;19:1123-1127. 
[12] Fernandez P, André V, Rieger J, et al. Nano-emulsion formation by emulsion phase inversion. Colloid Surf A-Physicochem Eng Asp. 2004;251:53-58.

[13] Choi AJ, Kim CJ, Cho YJ, et al. Characterization of capsaicin-loaded nanoemulsions stabilized with alginate and chitosan by self-assembly. Food Bioprocess Technol. 2011;4:1119-1126.

[14] Wang L, Li X, Zhang G, et al. Oil-in-water nanoemulsions for pesticide formulations. J Colloid Interface Sci. 2007;314:230-235.

[15] Kim JY, Lee MS, Jung S, et al. Anti-obesity efficacy of nanoemulsion oleoresin capsicum in obese rats fed a high-fat diet. Int J Nanomed. 2014;9:301-310.

[16] Lee MS, Kim CT, Kim IH, et al. Effects of capsaicin on lipid catabolism in 3T3-L1 adipocytes. Phytother Res. 2011;25:935-939.

[17] Rozen S, Skaletsky H. Primer3 on the WWW for general users and for biologist programmers. Methods Mol Biol. 2000;132:365-386.

[18] Choi AJ, Kim CJ, Cho YJ, et al. Effects of surfactants on the formation and stability of capsaicin-loaded nanoemulsions. Food Sci Biotechnol. 2009;18:1161-1172.

[19] Prusty D, Park BH, Davis KE, et al. Activation of MEK/ ERK signaling promotes adipogenesis by enhancing peroxisome proliferator-activated receptor $\gamma(\operatorname{PPAR} \gamma)$ and $\mathrm{C} /$ $\mathrm{EBPa}$ gene expression during the differentiation of 3T3-L1 preadipocytes. J Biol Chem. 2002;277:46226-46232.
[20] Spiegelman B. PPAR-gamma: adipogenic regulator and thiazolidinedione receptor. Diabetes. 1998;47:507-514.

[21] Thompson GM, Trainor D, Biswas C, et al. A highcapacity assay for PPAR $\gamma$ ligand regulation of endogenous aP2 expression in 3T3-L1 cells. Anal Biochem. 2004;330:21-28.

[22] Duplus E, Forest C. Is there a single mechanism for fatty acid regulation of gene transcription?. Biochem Pharmacol. 2002;64:893-901.

[23] Cao H, Sekiya M, Ertunc ME, et al. Adipocyte lipid chaperone AP2 is a secreted adipokine regulating hepatic glucose production. Cell Metab. 2013;17:768778.

[24] Feng Z, Haining Y, Xiaoman C, et al. Effect of yellow capsicum extract on proliferation and differentiation of 3T3-L1 preadipocytes. Nutrition (Burbank, Los Angeles County, Calif). 2014;30:319-325.

[25] Langin D, Holm C, Lafontan M. Adipocyte hormonesensitive lipase: a major regulator of lipid metabolism. Proc Nutr Soc. 1996;55:93-109.

[26] Li L, Wu L, Wang C, et al. Adiponectin modulates carnitine palmitoyltransferase-1 through AMPK signaling cascade in rat cardiomyocytes. Regul Pept. 2007;139:72-79.

[27] Ahn IS, Do MS, Kim SO, et al. Antiobesity effect of Kochujang (Korean fermented red pepper paste) extract in 3T3-L1 adipocytes. J Med Food. 2006;9:15-21. 